ALMEIDA, José Domingues de - Représentations du Français Langue Additionnelle à la FLUP. Para lá da tarefa: implicar os estudantes na aprendizagem de línguas estrangeiras no ensino superior. Porto: FLUP, 2019, pp. 12-24 DOI: https://doi.org/10.21747/9789898969217/paraa1

\title{
Représentations du Français Langue Additionnelle à la FLUP. Étude de cas et mise à jour didactique
}

\author{
José Domingues de Almeida \\ Faculdade de Letras da Universidade do Porto, Portugal, - APEF - \\ jalmeida@letras.up.pt
}

\begin{abstract}
Résumé
Nous partirons de considérations théoriques, critiques et statistiques pour dresser un état des lieux des représentations de la langue française au Portugal, notamment à partir de notre expérience, implication et responsabilité dans l'enseignement-apprentissage du Français Langue Additionnelle à la Faculté des Lettres de I'Université de Porto. En excursus, nous entendons recourir à un bref questionnaire pratique et direct aux étudiants en fin d'année académique afin de dégager des affects qui auraient une incidence didactique sur le déroulement et les résultats d'apprentissage.
\end{abstract}

Mots-clés: français, FLE, Portugal, FLUP, statistiques, représentations

\begin{abstract}
We will start from theoretical, critical and statistical considerations to draw up an inventory of representations of the French language in Portugal, in particular from our experience, involvement and responsibility in the teaching-learning of French as an Additional Language at the Faculty of Arts and Humanities - University of Porto. In excursus, we intend to resort to a short practical and direct questionnaire to the students at the end of the academic year in order to identify emotions that would have a didactic effect on the course and the learning outcomes.
\end{abstract}

Keywords: French, FFL, Portugal, FLUP, statistics, representations

Notre souci pour l'étude et le débat autour des représentations de la langue française au Portugal ne date pas d'aujourd'hui. En 2008, notre perspective était plutôt sombre et pessimiste puisqu'il fallait acter une allergie croissante des apprenants de français au Portugal. Et ce, notamment dans l'enseignement secondaire où une autre langue 
ALMEIDA, José Domingues de - Représentations du Français Langue Additionnelle à la FLUP. Para lá da tarefa: implicar os estudantes na aprendizagem de línguas estrangeiras no ensino superior. Porto: FLUP, 2019, pp. 12-24 DOI: https://doi.org/10.21747/9789898969217/paraa1

(l'espagnol) était entre-temps venue concurrencer le Français Langue Additionnelle (Almeida, 2008). Dans le cadre de l'Association Portugaise d'Études Françaises, tout un travail scientifique et pédagogique a été entrepris en partenariat avec l'Institut Français du Portugal afin de mieux cerner les tenants et aboutissants d'un malaise généralisé et systémique, souvent irrationnel, à l'endroit de la langue française.

À cet égard, nous avons pu suivre, décrire et décrypter l'évolution récente de cette tendance (Almeida, 2012, 2012a, 2016), laquelle pointe une certaine normalisation du statut du français chez nous, voire une surprenante récupération, une fois véritablement assumé le caractère appliqué et pragmatique de son usage non élitiste, non littéraire et surtout non hypostasié au Portugal, que l'on observe surtout à partir de la crise économique et financière des années 2010, laquelle a contraint beaucoup de nos concitoyens qualifiés à renouer dans l'urgence avec un usage pratique et utilitariste de cette langue dans un contexte migratoire d'un genre nouveau.

Les différentes prospections sur les représentations du français ont ceci en commun qu'elles mettent en évidence les facteurs affectifs de l'objet d'enseignementapprentissage appelé Français Langue Additionnelle, déjà mis en exergue par les études menées en neurosciences (Damásio, 1995, 1999) et la conception générale des aspects affectifs du discours scientifique en soi (Swain, 2013 ; Sander, 2015), lesquels montrent à l'envi combien l'affect ne s'oppose pas au cognitif. Bien au contraire, il le complète, voire l'explique (Damásio, 1995). En effet, l'entreprise de dégagement et d'interprétation des représentations affectives à l'endroit du français, notamment dans le contexte universitaire actuel, implique de saisir le rôle joué par les émotions représentationnelles dans le processus d'apprentissage (Dewaele, 2010).

Comme l'a abondamment souligné Simon Coffey, la projection, même en contexte d'enseignement-apprentissage dans une langue « autre », en l'occurrence le français, engage toujours la difficile, parce que non dite et latente, gestion affective de l'assomption de la distance affective entre le moi de l'apprenant et cette langue

“étrangère » (Coffey, 2015). C'est dire combien les aspects purement représentationnels interagissent et interfèrent dans cet apprentissage, et doivent être pris en compte en didactique du français, notamment à l'université.

Or la notion de « représentation » devient de plus en plus présente dans le champ des études portant sur les langues, leur appropriation et leur transmission. En fait, les représentations que les locuteurs ou les étudiants se font des langues qu'ils parlent ou apprennent, de leurs aspects normatifs, de leurs caractéristiques morphosyntaxiques ou phonétiques, ou de leurs statuts dans le cadre du schéma global du marché des langues 
ALMEIDA, José Domingues de - Représentations du Français Langue Additionnelle à la FLUP. Para lá da tarefa: implicar os estudantes na aprendizagem de línguas estrangeiras no ensino superior. Porto: FLUP, 2019, pp. 12-24 DOI: https://doi.org/10.21747/9789898969217/paraa1

et de la communication (Calvet, 1999) influent sur les outils et les stratégies qui seront déployés et mis en œuvre aussi bien pour leur enseignement-apprentissage que pour leur utilisation concrète (Dabène, 1997).

Bien évidemment, ces coefficients affectifs échappent aux descripteurs habituels du Cadre Européen Commun de Référence pour les Langues (2001) dans la mesure où ils ne visent pas la réalisation d'une quelconque «tâche» selon la perspective actionnelle qui régit ledit document, et qu'ils s'intéressent à des aspects somme toute subsidiaires et apparemment secondaires de l'enseignement-apprentissage des langues. En quelque sorte, nous sommes ici en-deçà et au-delà de la tâche, peut-être à côté, mais toujours dans l'optique de cet apprentissage.

L'importance didactique de l'attention portée aux représentations des langues a été pertinemment soulignée, d'une part, par les études théoriques des représentations en soi et, d'autre part, par l'application pratique de ces approches sur des univers ou des échantillons bien délimités et diversifiés. Dans le premier cas, force est d'évoquer les travaux théoriques entrepris par Véronique Castellotti et Danièle Moore. Comme ces deux auteures le rappellent, cette notion est avant tout redevable aux recherches en psychologie sociale (Moscovici, 1989; Castellotti \& Moore,2002, p. 8), c'est-à-dire à l'approche des représentations sociales en tant que « forme[s] de connaissance socialement élaborée[s] et partagée[s], ayant une visée pratique et concourant à la construction d'une réalité commune à un ensemble social » (Jodelet, 1989), lesquelles fonctionnent sur la base des principes ou processus successifs d'objectivation et d'ancrage (Moscovici, 1961). Aussi les représentations sociales sont-elles «Collectivement produites et engendrées» (Moscovici, 1994, pp. 62-85) et résultent-elles d'une «élaboration collective et consensuelle au sein d'un groupe social» (Herrera, 2011, p. 59).

Par ailleurs, l'étude de ces représentations suppose que l'on considère de façon critique la validité de certains stéréotypes associés à l'usage et à l'apprentissage d'une langue additionnelle. Or, comme le soulignent Castellotti et Moore,

Les stéréotypes identifient des images stables et décontextualisées, schématiques et raccourcies, qui fonctionnent dans la mémoire commune, et auxquelles adhèrent certains groupes. Le degré d'adhésion et de validité que leur portent certains groupes de locuteurs ou des individus peuvent être liés à des conduites, à des comportements linguistiques et à des comportements d'apprentissage (Castellotti \& Moore,2002, p. 8). 
ALMEIDA, José Domingues de - Représentations du Français Langue Additionnelle à la FLUP. Para lá da tarefa: implicar os estudantes na aprendizagem de línguas estrangeiras no ensino superior. Porto: FLUP, 2019, pp. 12-24 DOI: https://doi.org/10.21747/9789898969217/paraa1

En outre, force est de reconnaître que bien des enquêtes sur les représentations de la langue française, notamment en milieu universitaire, ont été menées dernièrement un peu partout dans le monde (Mexique, Algérie, Palestine, Belgique néerlandophone), dont les résultats font l'objet de publications, voire de dissertations (Medoukh, 2008;Djeghar, 2009; Delahaie, 2010; Herrera, 2011). Elles sont fondées sur des questionnaires brefs, simples, directs, parfois rudimentaires. Même si elles s'avèrent valables d'un point de vue affectif, elles manquent souvent de pertinence dans la complexification et dans la problématicité des questions posées.

Pour notre part, nous avons mené une enquête similaire, certes, dans le propos de dégager des représentations spécifiques sur la langue française en tant qu'objet d'enseignement-apprentissage à la Faculté des Lettres de l'Université de Porto en 2018, mais en cohérence avec les points programmatiques des unités de cours que nous assurons dans le cursus des études françaises: «linguistique française», «littératures francophones» et «francophonie et mondialisation». En fait, il s'agissait surtout de sonder les étudiants de français à partir de problématiques qui se trouvent inévitablement en-deçà et au-delà de la tâche, pas toujours faciles à verbaliser, mais qui s'invitent forcément dans l'enseignement-apprentissage de la langue comme, par exemple, l'insécurité linguistique (Klinkenberg, 1989), la surconscience linguistique (Gauvin,1997), les aspects émotifs et affectifs, les représentations immédiates ou réfléchies, etc.

Notre méthodologie a consisté en la mise en ligne d'un questionnaire à partir du logiciel Google Forms, intitulé «Représentations de la langue française chez les étudiants de la FLUP », comprenant dix questions, dont trois ouvertes et sept fermées (oui / non / peut-être ou éventail prédéterminé de réponses) directes et bien ciblées. Ceci a permis aux étudiants de répondre assez rapidement au questionnaire (cinq minutes suffisaient largement) à une période de l'année académique où ils ne se trouvaient plus vraiment pris par les cours et pas encore par les examens, et où ils avaient inévitablement une conscience plus solide de leur rapport à la langue française. II est sous-entendu que le fait que les répondants anonymes à ce questionnaire sont des étudiants en études françaises n'implique pas forcément une approche moins problématisante de la langue d'apprentissage. Le questionnaire était disponible, et a été administré sur internet, entre le 11 mai et le 03 juin 2018, et a obtenu trente et une (31) réponses. Quoique le nombre de réponses ne soit pas très élevé par rapport à l'univers des étudiants de français de la FLUP (près de 300 étudiants), l'échantillon n'en est pas moins représentatif et valable. 
ALMEIDA, José Domingues de - Représentations du Français Langue Additionnelle à la FLUP. Para lá da tarefa: implicar os estudantes na aprendizagem de línguas estrangeiras no ensino superior.

Les dix questions étaient:

1. "Quand vous pensez au, parlez français, quelles représentations associezvous instinctivement à l'usage de cette langue? »;

2. «Trouvez-vous le français une langue particulièrement élitiste?»;

3. «Trouvez-vous le français une langue particulièrement difficile?»;

4. «Quels stéréotypes associez-vous spontanément à l'usage du français?»;

5. «Dans quel(s) objectif(s) concret(s) apprenez-vous le français?»;

6. «Quel(s) autre(s) contact(s) avez-vous avec le français en dehors du contexte académique?»;

7. «Considérez-vous le français une langue agréable à parler / entendre parler $? »$;

8. «Pensez-vous que le français a une vocation à la communication en contexte global ?»;

9. «Quand vous parlez français, avez-vous peur de vous tromper ; éprouvez-vous un sentiment d'insécurité que vous n'éprouvez pas avec une autre langue étrangère?»;

10. "Quand vous parlez français, vous vous sentez ...».

Les résultats au questionnaire ont été les suivants:

À la question ouverte 1, 24 (vingt-quatre) étudiants ont suggéré des éléments assez disparates: liberté, égalité, fraternité; le français, une langue très similaire au portugais ; l'élégance, les villes françaises, notamment Paris; démocratie, humanisme, valorisation et promotion de la culture (francophonie); culture, chic, connaissance; Francophonie; l'intelligence, la séduction et la musicalité; les organisations internationales; la formalité des situations; le romantisme, l'éthique et Versailles; le passé prestigieux et le statut politique; la monarchie et le rôle et mission de la France dans le contexte international; le voyage et le tourisme; I'harmonie de la langue, voire aucune représentation (langue tout simplement apprise).

\section{À la question fermée 2, 31 (trente et un) étudiants ont répondu:}


ALMEIDA, José Domingues de - Représentations du Français Langue Additionnelle à la FLUP. Para lá da tarefa: implicar os estudantes na aprendizagem de línguas estrangeiras no ensino superior.

Trouvez-vous le français une langue particulièrement élitiste?

31 respostas

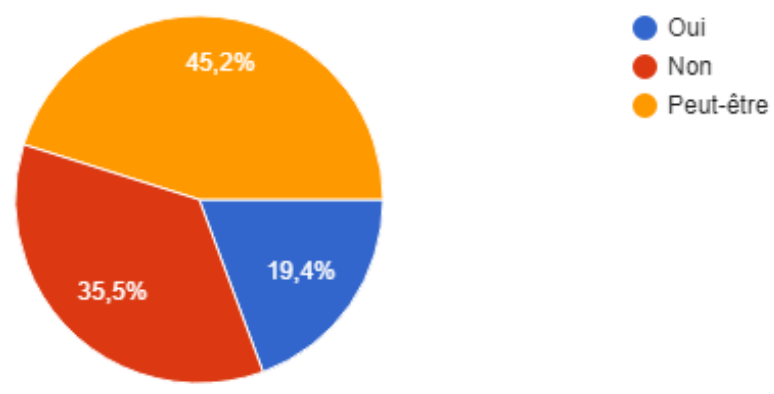

\section{À la question fermée 3, 31 (trente et un) étudiants ont répondu}

Trouvez-vous le français une langue particulièrement difficile? 31 respostas
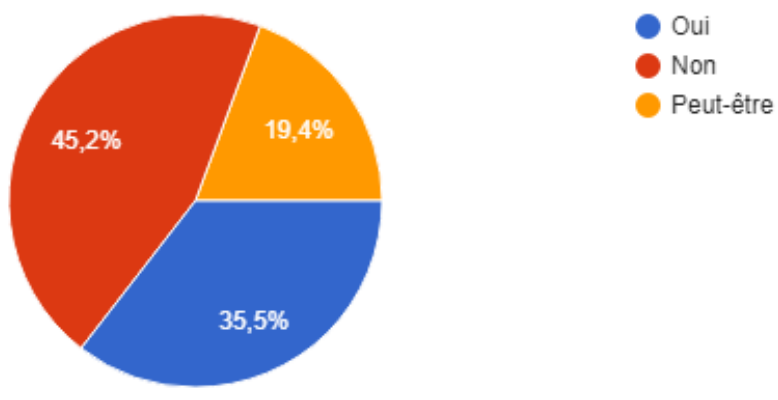

\section{À la question ouverte 4, 24 (vingt-quatre) étudiants ont suggéré}

les éléments hétéroclites suivants - qui ne se réfèrent pas toujours à des clichés et qui rejoignent parfois inévitablement le sens de la question 1 : le romantisme; la langue de l'amour et de la bonne cuisine (gastronomie) ; la moue pour parler français ; l'élitisme; un peuple fier de ses traditions et de sa culture, et la langue de la diplomatie; des élites politiques et économiques, des références littéraires, ainsi que le fait que plusieurs mots français soient empruntés dans un registre soutenu par d'autres langues; le cliché du garçon en béret dans un pull rayé et une baguette; les sons nasaux; des gens arrogants; le croissant, la baguette; le chic, le prestige et l'éloquence; l'incompréhension des Français devant nos fautes de français ; la culture intellectuelle; une langue délicate; la difficulté d'apprentissage (conjugaison et orthographe); voire aucun stéréotype du tout. 
ALMEIDA, José Domingues de - Représentations du Français Langue Additionnelle à la FLUP. Para lá da tarefa: implicar os estudantes na aprendizagem de línguas estrangeiras no ensino superior.

\section{À la question ouverte $\mathbf{5}$ sur les objectifs d'apprentissage, 28 (vingt-huit)}

étudiants ont répondu : opportunités d'emploi; la beauté et l'utilité de la langue; métier du tourisme; langue des organismes internationaux, notamment l'ONU; ouverture sur une autre langue; aspiration au plurilinguisme; développement culturel et littéraire; la carrière d'enseignant ou de traducteur.

À la question fermée 6 sur les contextes de contact avec le français, 31 (trente et un) étudiants ont répondu:

Quel(s) autre(s) contact(s) avez-vous avec le français en dehors du contexte académique?

31 respostas
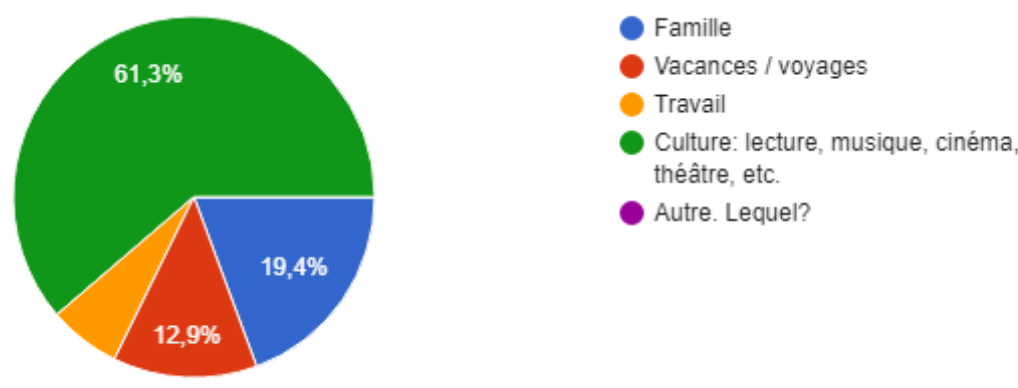

À la question fermée 7 sur l'appréciation esthétique de la langue française, 30 (trente) étudiants ont répondu :

Considérez-vous le français une langue agréable à parler / entendre parler?
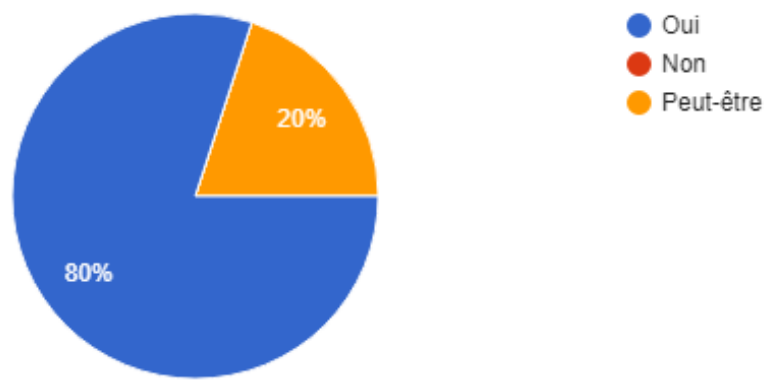
ALMEIDA, José Domingues de - Représentations du Français Langue Additionnelle à la FLUP. Para lá da tarefa: implicar os estudantes na aprendizagem de línguas estrangeiras no ensino superior. Porto: FLUP, 2019, pp. 12-24 DOI: https://doi.org/10.21747/9789898969217/paraa1

À la question fermée 8 sur la vocation globale de la langue française, 29 (vingt-neuf) étudiants ont répondu :

Pensez-vous que le français a une vocation à la communication en contexte global?

29 respostas

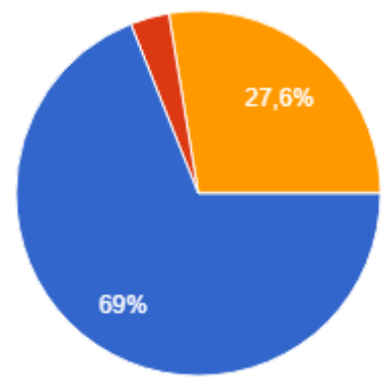

$$
\begin{aligned}
& \text { Oui } \\
& \text { Non } \\
& \text { Peut-être }
\end{aligned}
$$

À la question fermée 9 sur le sentiment d'insécurité linguistique en Français Langue Additionnelle, 31 (trente et un) étudiants ont répondu:

Quand vous parlez français, avez-vous peur de vous tromper ; éprouvez-vous un sentiment d'insécuri... pas avec une autre langue étrangère? 31 respostas

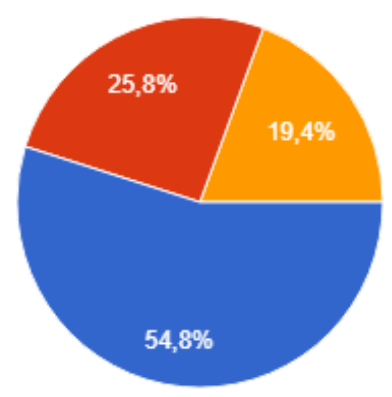


ALMEIDA, José Domingues de - Représentations du Français Langue Additionnelle à la FLUP. Para lá da tarefa: implicar os estudantes na aprendizagem de línguas estrangeiras no ensino superior.

\section{À la question fermée 10 sur les attributs qualitatifs de l'usage de langue française, 31 (trente et un) étudiants ont répondu :}

\section{Quand vous parlez français, vous vous sentez \\ 31 respostas}
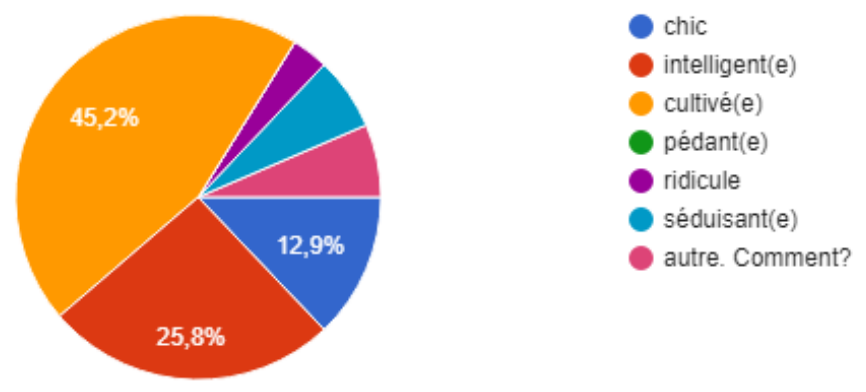

II ressort de cette enquête que certains clichés autour du Français Langue Additionnelle ont la vie dure. Les représentations sociales de la langue française, même en contexte d'apprentissage universitaire, ont du mal à évoluer: le chic, l'élégance, l'art de vivre et la baguette demeurent des éléments stéréotypés, comme le montrent à l'envi les réponses aux questions ouvertes 1 et 4 . Cependant, il appert que les étudiants ont nettement des perspectives appliquées, professionnelles et pragmatiques de la langue. Outre la traduction et l'enseignement, il y a le tourisme, la diplomatie et l'employabilité en général.

Ceci dit, à la question 4, les répondants affirment entretenir un certain contact régulier avec des manifestations culturelles en français, notamment le cinéma et la chanson, alors que son utilité communicationnelle globale est amplement reconnue, à la question fermée 8 , par $69 \%$ des opinions.

Si le caractère élitiste du français, bien que non majoritairement exprimé, est largement rappelé (question 2), l'évaluation que les étudiants se font du degré général de difficulté du français est assez nuancée. En effet, l'indécision est assez importante, même à l'endroit d'une langue étrangère dont on a fait quand même le choix délibéré de l'apprentissage. Ceci étant, le ressenti de l'insécurité et de l'inhibition linguistiques demeure assez fort $(54,8 \%$ des répondants à la question fermée 9$)$.

Quoi qu'il en soit, le caractère esthétique de la langue française obtient une forte majorité des réponses ( $80 \%$ à la question fermée 7$)$, tout comme les attributs représentationnels et les connotations spontanément associés à l'usage public du français. En effet, les réponses à la question fermée 10 révèlent des sentiments très 
ALMEIDA, José Domingues de - Représentations du Français Langue Additionnelle à la FLUP. Para lá da tarefa: implicar os estudantes na aprendizagem de línguas estrangeiras no ensino superior. Porto: FLUP, 2019, pp. 12-24 DOI: https://doi.org/10.21747/9789898969217/paraa1

cohérents autour d'images socialement positives et mélioratives du français. Si 45,2\% affirment se sentir «cultivés», $25,8 \%$ se disent « intelligents » et $12,9 \%$ «séduisants».

II ne suffit pas de prendre acte des réponses à ce questionnaire. II s'agit d'y apporter des solutions didactiques. Or les enquêtes que nous avons signalées plus haut n'étaient pas vraiment concluantes ou ne creusaient pas les raisons en amont des conclusions tirées. Certaines représentations proviennent inévitablement de la transmission de clichés par les médias. Mais d'autres sont reproduites et re-légitimées dans les manuels de FLE au niveau de l'enseignement secondaire. Chez nous, certaines études ont mis en exergue la résistance des clichés culturels dans l'élaboration des manuels de FLE et ont pointé plusieurs solutions (Almeida, 2014).

Celles-ci passent par une « dés-hexagonalisation » des références présentes dans les manuels, notamment au niveau des contenus, de la toponymie, de l'onomastique et des aspects culturels, qu'ils soient patrimoniaux, gastronomiques ou purement représentationnels. Or ces aspects surabondent dans les manuels et dans les matériels didactiques, et ce en dépit de la globalisation croissante aussi bien du fait linguistique que culturel francophone. C'est dire que l'on fait encore souvent comme si la mondialisation n'était pas passée par la francosphère (Wolton, 2006), comme si le centre franco-parisien produisait et mobilisait à lui seul les affects représentationnels du vivre et du parler en français, comme si la langue française (et ses représentations habituelles) n'était pas dé-nationalisable, ce dont d'aucuns ont pris acte dans le fait littéraire francophone (Le Bris \& Rouaud, 2007).

À l'aune de ce qui vient d'être considéré et des résultats de notre modeste questionnaire, il s'agirait en classe de Français Langue Additionnelle en contexte universitaire, et dans les périphéries de la tâche, de promouvoir une communication décomplexée et déculpabilisante en français, où on commettrait des fautes, où on pourrait même en rire, mais qui permettrait de renouer avec la production orale noninhibitoire. II faut dépasser les stéréotypes d'élitisme et de difficulté. II est essentiel de rendre au français son potentiel communicationnel en classe, d'autant plus qu'une écrasante majorité des étudiants croit à la vocation globale de cette langue. Pour ce, les classes ne devraient pas dépasser un effectif de vingt à vingt-cinq étudiants pour favoriser l'échange et la mise en confiance. On gagnerait beaucoup à multiplier les registres de langage dans les matériels didactiques ou documents didactisés afin de rendre une image moins normée ou élitiste de la langue. Ceci passe aussi par une plus grande flexibilité au niveau de l'oral et de l'orthographe, en tenant compte de la possibilité de différents accents et des réformes orthographiques qui ont été lancées, 
ALMEIDA, José Domingues de - Représentations du Français Langue Additionnelle à la FLUP. Para lá da tarefa: implicar os estudantes na aprendizagem de línguas estrangeiras no ensino superior. Porto: FLUP, 2019, pp. 12-24 DOI: https://doi.org/10.21747/9789898969217/paraa1

voire en allant plus loin là où cela s'avère utile, notamment dans la morphosyntaxe, comme on le voit dans le débat sur l'accord du participe passé avec l'auxiliaire «avoir». Dans tous les cas, il faut absolument jouer sur l'intercompréhension entre langues romanes, mais aussi sur les affinités historiques entre le français et l'anglais par le biais de points didactiques en linguistique diachronique.

En outre, des activités para-curriculaires doivent être sérieusement envisagées: clubs de lecture, de presse, commentaires de films, débats, blogues, réseaux sociaux, etc. L'actualité en français est une abondante source de contenus et de références qui reconstruisent et restructurent les représentations. En fait, il ne faut pas jeter le bébé avec l'eau du bain. Si la plupart des étudiants se sentent cultivés quand ils s'expriment en français, cet « affect » est lui aussi positif. II se voit même fortement relayé par le fait qu'une étonnante majorité de nos étudiants aime (entendre) parler notre langue, aspect affectif s'il en est, qui se place au-delà de la tâche et qui la facilite.

\section{Références bibliographiques}

Almeida, J. Domingues de (2008). Que reste-t-il de nos amours? Quelques réflexions sur le statut de la langue française au Portugal aujourd'hui en guise de mise au point et de stratégie didactique. Çédille, 4, 33-44: En ligne http://webpages.ull.es/users/cedille/cuatro/almeida.pdf [consulté le 23/01/2019]

Almeida, J. Domingues de (2012). Le français, une langue « étrangère » dans le village global ? Perspectives et attentes d'une politique linguistique du français en contexte mondialisé. La Clé des Langues,1-8.

Almeida, J. Domingues de (2012a). Argumentaire pour le français au Portugal et orbi. Arguments, apories et représentations. Carnets, nº especial outono-inverno 2011-12 «Invasions \& Évasions françaises. La France et nous, nous et la France», 347-356. En ligne: https://journals.openedition.org/carnets/8018_[consulté le 23/01/2019]

Almeida, J. Domingues de (2014). De l'éducation à la citoyenneté au socialement correct: tour d'horizon du millésime 2012 (manuels de $7^{\mathrm{e}} \mathrm{FLE}$ ). Intercâmbio, 7, 2, 74-83.

Almeida, J. Domingues de (2016). Présence actuelle du français au Portugal. Entre rayonnement et allergie: la quête d'une place introuvable. Présence et interférences franco-ibériques. Langue, littérature et culture. In Mํㅡ. T. G. Castanyer, L. Llecha-Llop García \& Alicia P. Desvaux (Eds.), Berne: Peter Lang, 11-23.

Calvet, L.-J. (1999). Pour une écologie des langues du monde. Paris: Plon. 
ALMEIDA, José Domingues de - Représentations du Français Langue Additionnelle à la FLUP. Para lá da tarefa: implicar os estudantes na aprendizagem de línguas estrangeiras no ensino superior. Porto: FLUP, 2019, pp. 12-24 DOI: https://doi.org/10.21747/9789898969217/paraa1

Castelloti, V. \& Moore, D. (2002). Représentations sociales des langues et enseignements. Guide pour l'élaboration des politiques linguistiques éducatives en Europe, Guide pour l'élaboration des politiques linguistiques éducatives en Europe - De la diversité linguistique à l'éducation plurilingue. Strasbourg: Conseil de l'Europe.

Coffey, S. (2015). Rendre le banal spécial à travers l'apprentissage des langues. Le langage et l'homme L1, 71-84.

Conseil de l'Europe. (2001). Cadre Européen Commun de Référence pour les Langues. Paris: Didier.

Dabène, L. (1997). L'image des langues et leur apprentissage. In Matthey, M (Ed.). Les langues et leurs images. Lausanne: Loisirs Et Pédagogie. 19-23.

Damásio, A. (1995). L'erreur de Descartes: la raison des émotions. Paris: Odile Jacob.

Damásio, A. (1999). Le sentiment de soi. Corps, émotions, conscience. Paris: Odile Jacob.

Delahaie, J. (2010). La didactique plurilingue et pluriculturelle dans un pays multilingue: le cas de l'enseignement du FLE en Belgique néerlandophone. En ligne: https://www.researchgate.net/publication/280816901_La_didactique_plurilingue_et_plu riculturelle_dans_un_pays_multilingue_le_cas_de_l'enseignement_du_FLE_en_Belgiq ue_neerlandophone [consulté le 23/01/2019]

Djeghar, A. (2009). Les représentations linguistiques et culturelles dans l'enseignement / apprentissage du français langue étrangère. Synergies Algérie, 5, 191 198.

Dewaele, J-M. (2010). Emotions in Multiple Languages. Basingstoke: Palgrave / Macmillan.

Gauvin, L. (1997). D'une langue, l'autre. La surconscience linguistique de l'écrivain francophone. L'écrivain francophone à la croisée des langues. Paris: Karthala. 5-15.

Jodelet, D. (1989). Les représentations sociales. Paris: PUF.

Klinkenberg, J.-M. (1989). Le problème de la langue d'écriture dans la littérature francophone de Belgique de Verhaeren à Verheggen. In A. Vigh (Éd.). L'ldentité culturelle dans les littératures de langue française. Actes du Colloque de Pécs. Paris: P. V. Pécs / ACCT. 69-73.

Le Bris, M. \& Rouaud, J. (2007). Pour une littérature-monde. Paris: Gallimard.

Medoukh, Z. (2008). Les représentations du français chez les étudiants palestiniens. Production des enseignants et des chercheurs. En ligne: http://eprints.aidenligne- 
ALMEIDA, José Domingues de - Représentations du Français Langue Additionnelle à la FLUP. Para lá da tarefa: implicar os estudantes na aprendizagem de línguas estrangeiras no ensino superior.

francais-universite.auf.org/24/1/pdf_Ziad_Medoukh_Representatio.pdf. [consulté le 23/01/2019]

Moscovici, S. (1961). La psychanalyse, son image, son public. Paris: PUF.

Moscovici, S. (1989). Les représentations sociales. Paris: PUF.

Moscovici, S. (1994). Les représentations collectives aux représentations sociales. Les représentations sociales. Paris: PUF. 62-86.

Swain, M. (2013). The inseparability of cognition and emotion in second language learning. Cambridge: Cambridge University Press.

Sander, D. (2015). Le monde des émotions. Paris: Belin.

Velázquez, Herrera A. (2011). Représentations sociales de la langue française et motivations de son apprentissage: enquête auprès des étudiants mexicains spécialistes et non-spécialistes. Synergies Mexique, 1, 57-69.

Wolton, D. (2006). Demain la francophonie. Paris: Flammarion. 